\title{
Stringy Gravity: Solving the Dark Problems at 'short' distance
}

\author{
Jeong-Hyuck Park ${ }^{\star}$ \\ ${ }^{1}$ Department of Physics, Sogang University, 35 Baekbeom-ro, Mapo-gu, Seoul 04107, Korea \\ ${ }^{2}$ Center for Theoretical Physics of the Universe, Institute for Basic Science (IBS), Seoul 08826, Korea
}

\begin{abstract}
Dictated by Symmetry Principle, string theory predicts not General Relativity but its own gravity which assumes the entire closed string massless sector to be geometric and thus gravitational. In terms of $R /(M G)$, i.e. the dimensionless radial variable normalized by mass, Stringy Gravity agrees with General Relativity toward infinity, but modifies it at short distance. At far short distance, gravitational force can be even repulsive. These may solve the dark matter and energy problems, as they arise essentially from small $R /(M G)$ observations: long distance divided by much heavier mass. We address the pertinent differential geometry for Stringy Gravity, stringy Equivalence Principle, stringy geodesics and the minimal coupling to the Standard Model. We highlight the notion of 'doubled-yet-gauged' coordinate system, in which a gauge orbit corresponds to a single physical point and proper distance is defined between two gauge orbits by a path integral.
\end{abstract}

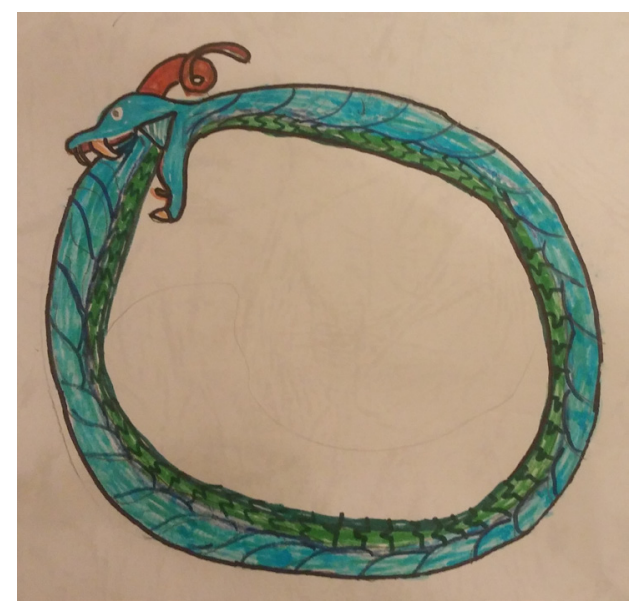

Uroboros: An ancient Egyptian symbol for a serpent which eats its own tail

^e-mail: park@sogang.ac.kr 


\section{Introduction}

Ever since Einstein formulated his theory of gravity, i.e. General Relativity (GR), the metric, $g_{\mu v}$, has been privileged to be the only geometric and hence gravitational field, on account of the adopted Riemannian geometry. All other fields or particles are meant to be 'extra' matters. In particular, the Riemannian metric sets a proper length which every free particle would minimize, following the geodesic,

$$
\ddot{x}^{\lambda}+\left\{\begin{array}{c}
\lambda \\
\mu \nu
\end{array}\right\} \dot{x}^{\mu} \dot{x}^{v}=0
$$

The gravitational coupling to matters, or to the Standard Model, is 'minimally' fixed by the metric and covariant derivatives which ensure both diffeomorphisms and local Lorentz symmetry. In the words of Cheng-Ning Yang, symmetry dictates interaction.

On the other hand, string theory suggests us to put a two-form gauge potential, $B_{\mu \nu}$, and a scalar dilaton, $\phi$, on an equal footing along with the metric. Forming the massless sector of closed strings, they are ubiquitous in all string theories, with the conventional (Riemannian) description given by

$$
\int \mathrm{d}^{D} x \sqrt{-g} e^{-2 \phi}\left(R_{g}+4 \partial_{\mu} \phi \partial^{\mu} \phi-\frac{1}{12} H_{\lambda \mu \nu} H^{\lambda \mu \nu}\right) \quad \text { where } \quad H=\mathrm{d} B .
$$

Furthermore, a genuine stringy symmetry, called T-duality, can mix three of them $[1,2]$. These may well hint at the existence of Stringy Gravity which should take the entire closed string massless sector to be geometric and gravitational. For this, a novel differential geometry beyond Riemann is required, in particular to shed light on the $\mathbf{O}(D, D)$ T-duality symmetry hidden in the above action.

After series of pioneering works on 'doubled sigma models' [3-8] and 'double field theory' (DFT) [9-13] (c.f. [14-16]), such an idea of Stringy Gravity has materialized. The word 'double' refers to the fact that doubled $(D+D)$-dimensional coordinates are used for the description of $D$ dimensional physical spacetime. While such a usage was historically first made in the case of a torus background - by introducing a dual coordinate conjugate to the string winding momentum - the doubled coordinates turned out to be far more general: they can be applied to any compact or non-compact spacetime, as well as to not only string but also to particle physics.

\section{Stringy Gravity: Uroboros solution to Dark Problems}

\subsection{Stringy differential geometry}

Stringy Gravity of our interest adopts the doubled-yet-gauged coordinate system [17] which meets two properties. Firstly, an $\mathbf{O}(D, D)$ group is a priori postulated, having the constant invariant "metric",

$$
\mathcal{J}_{A B}=\left(\begin{array}{ll}
\mathbf{0} & \mathbf{1} \\
\mathbf{1} & \mathbf{0}
\end{array}\right)
$$

With the inverse, $\mathcal{J}^{A B}$, it can freely raise and lower the $\mathbf{O}(D, D)$ vector indices (capital letters, $A, B, \cdots)$. Secondly, the doubled coordinates are gauged by an equivalence relation,

$$
x^{A} \sim x^{A}+\Delta^{A}(x)
$$

where $\Delta^{A}$ is an arbitrary 'derivative-index-valued' vector. This means that its superscript index must be identifiable as that of derivative, $\partial^{A}=\mathcal{J}^{A B} \partial_{B}$. For example, with arbitrary functions, $\Phi_{i}$, belonging to the theory, $\Delta^{A}=\sum_{i, j} \Phi_{i} \partial^{A} \Phi_{j}$. The equivalence relation (4) can be realized by requiring that every 
field or function in Stringy Gravity - such as $\Phi_{i}$ itself, physical fields, local symmetry parameters and their arbitrary derivatives - should be invariant under the coordinate gauge symmetry shift, ${ }^{1}$

$$
\Phi_{k}(x+\Delta)=\Phi_{k}(x) \quad \Longleftrightarrow \quad \Delta^{A} \partial_{A}=0 .
$$

In this way, it becomes not a point but a gauge orbit in the doubled coordinate system that corresponds to a single physical point. The above coordinate gauge symmetry invariance is equivalent to so called the 'section condition' in DFT,

$$
\partial_{A} \partial^{A}=0 .
$$

With respect to the off block-diagonal form of the $\mathbf{O}(D, D)$ metric (3), the doubled coordinates split into two parts: $x^{A}=\left(\tilde{x}_{\mu}, x^{\nu}\right)$, and hence $\partial_{A} \partial^{A}=2 \tilde{\partial}^{\mu} \partial_{\mu}$. The general solution to the section condition is then given by $\tilde{\partial}^{\mu} \equiv 0$, up to $\mathbf{O}(D, D)$ rotations $[10,11]$.

General covariance in doubled-yet-gauged spacetime, or doubled diffeomorphisms, are given by

$$
\delta x^{A}=V^{A}, \quad \delta \partial_{A}=-\partial_{A} V^{B} \partial_{B}=\left(\partial^{B} V_{A}-\partial_{A} V^{B}\right) \partial_{B},
$$

and for a covariant tensor (or tensor density with weight $\omega$ ), ${ }^{2}$

$$
\delta T_{A_{1} \cdots A_{n}}=-\omega \partial_{B} V^{B} T_{A_{1} \cdots A_{n}}+\sum_{i=1}^{n}\left(\partial_{B} V_{A_{i}}-\partial_{A_{i}} V_{B}\right) T_{A_{1} \cdots A_{i-1}{ }^{B}{ }_{A_{i+1} \cdots A_{n}}} .
$$

The whole massless sector of closed strings, or stringy gravitons, consist of a unit-weighted scalar density given by an exponentiation of DFT-dilaton, $e^{-2 d}$, and a symmetric projector,

$$
P_{A B}=P_{B A}, \quad P_{A}^{B} P_{B}^{C}=P_{A}^{C},
$$

which accompanies the complementary, orthogonal projector, $\bar{P}_{A B}=\mathcal{J}_{A B}-P_{A B}$. It follows that the difference of the two projectors sets a symmetric $\mathbf{O}(D, D)$ element, i.e. DFT-metric, $P_{A B}-\bar{P}_{A B}=\mathcal{H}_{A B}$, satisfying $\mathcal{H}_{A B}=\mathcal{H}_{B A}$ and $\mathcal{H}_{A}{ }^{C} \mathcal{H}_{B}{ }^{D} \mathcal{J}_{C D}=\mathcal{J}_{A B}$.

The above $\mathbf{O}(D, D)$ covariant defining properties of the stringy gravitons can be conventionally solved by $\left\{g_{\mu \nu}, B_{\mu \nu}, \phi\right\}$. However, not all the solutions can be parametrized by these GR variables. Stringy Gravity is more general than GR: it encompasses 'non-Riemannian' geometries where the Riemannian metric, $g_{\mu \nu}$, cannot be defined, even locally (see [18-20] for examples and [21] for the complete classification and relations to Newton-Cartan/Carroll gravities, chiral strings etc.).

Requiring compatibility with all the stringy gravitons, i.e. $\nabla_{A} d=0, \nabla_{A} P_{B C}=0, \nabla_{A} \bar{P}_{B C}=0$, and imposing some torsionless conditions, one can uniquely determine the connection for a diffeomorphism covariant derivative, $\nabla_{A}=\partial_{A}+\Gamma_{A}[22]$,

$$
\begin{aligned}
\Gamma_{C A B}= & 2\left(P \partial_{C} P \bar{P}\right)_{[A B]}+2\left(\bar{P}_{[A}^{D} \bar{P}_{B]}^{E}-P_{[A}^{D} P_{B]}^{E}\right) \partial_{D} P_{E C} \\
& -4\left(\frac{1}{P_{M^{M}-1}} P_{C[A} P_{B]}^{D}+\frac{1}{\bar{P}_{M^{M}-1}} \bar{P}_{C[A} \bar{P}_{B]}^{D}\right)\left(\partial_{D} d+\left(P \partial^{E} P \bar{P}\right)_{[E D]}\right) .
\end{aligned}
$$

This stringy analogue of the Christoffel symbol constitutes subsequently a scalar curvature, $S_{(0)}$, and a two-indexed "Ricci" curvature, $P_{A}{ }^{C} \bar{P}_{B}{ }^{D} S_{C D}$, which further leads to the stringy counterpart of the conserved Einstein tensor: $G_{A B}=4 P_{[A}{ }^{C} \bar{P}_{B]}{ }^{D} S_{C D}-\frac{1}{2} \mathcal{J}_{A B} S_{(0)}$ satisfying $\nabla_{A} G^{A B}=0$ [23].

The scalar curvature multiplied by the unit-weighted integral measure, $e^{-2 d} S_{(0)}$, sets then the action for pure Stringy Gravity, which agrees with (2) upon Riemannian parametrization and up to total derivatives. Its equations of motion amount to the vanishing of the stringy Einstein tensor, $G_{A B}=0$, or equivalently $S_{(0)}=0$ and $P_{A}{ }^{C} \bar{P}_{B}{ }^{D} S_{C D}=0$. In particular, constant background solutions include not only the flat Minkowskian spacetime but also non-Riemannian ones, e.g. $\mathcal{H}_{A B}=\mathcal{J}_{A B}$, etc. [21].

\footnotetext{
${ }^{1}$ See also Eq.(13) for the cases where the doubled coordinates are dynamical fields.

${ }^{2}$ Eq.(8) corresponds to the passive counterpart of "generalized Lie derivative" à la Siegel [10].
} 


\subsection{Minimal coupling to the Standard Model}

To couple to fermions, it is necessary to introduce a pair of vielbeins, $V_{A p}, \bar{V}_{A \bar{p}}$, which form the mutually orthogonal projectors, $P_{A B}=V_{A p} V_{B}{ }^{p}, \bar{P}_{A B}=\bar{V}_{A \bar{p}} \bar{V}_{B} \bar{p}$, and satisfy their own defining properties:

$$
V_{A p} V_{q}^{A}=\eta_{p q}, \quad \bar{V}_{A \bar{p}} \bar{V}_{\bar{q}}^{A}=\bar{\eta}_{\bar{p} \bar{q}}, \quad V_{A p} \bar{V}^{A \bar{p}}=0, \quad V_{A p} V_{B}{ }^{p}+\bar{V}_{A \bar{p}} \bar{V}_{B}^{\bar{p}}=\mathcal{J}_{A B} .
$$

The unbarred and barred lowercase letters are the vector indices of twofold local Lorentz symmetries, $\operatorname{Spin}(1, D-1)$ and $\operatorname{Spin}(D-1,1)$, subject to Minkowskian metrics, $\eta_{p q}$ (mostly plus) and $\bar{\eta}_{\bar{p} \bar{q}}$ (mostly minus), respectively. The vielbeins diagonalize $\mathcal{J}_{A B}$ and $\mathcal{H}_{A B}$ simultaneously, with the eigenvalues, $(\eta,+\bar{\eta})$ and $(\eta,-\bar{\eta})$. Physically, the doubling of the spin groups implies a pair of locally inertial frames which exist separately for the left and right closed string modes $[24]{ }^{3}$ Now, to take care of all the local symmetries in Stringy Gravity, the covariant derivative generalizes to $\nabla_{A}=\partial_{A}+\Gamma_{A}+\Phi_{A}+\bar{\Phi}_{A}$, where the last two are $\operatorname{Spin}(1, D-1)$ and $\operatorname{Spin}(D-1,1)$ connections. These are fixed by the compatibility with the vielbeins, $\nabla_{A} V_{B p}=0, \nabla_{A} \bar{V}_{B \bar{p}}=0$, and thus related to the stringy Christoffel symbol (10) [26].

With the basics of the stringy differential geometry, $\left\{d, V_{A p}, \bar{V}_{A \bar{p}}, \nabla_{A}, S_{(0)}\right\}$, the Standard Model can be straightforwardly, and minimally, coupled to Stringy Gravity [27]. In particular, fermionic kinetic terms read

$$
e^{-2 d} \bar{\psi} \gamma^{A} \nabla_{A} \psi=e^{-2 d} \bar{\psi} V_{p}^{A} \gamma^{p}\left(\partial_{A} \psi+\frac{1}{4} \Phi_{A p q} \gamma^{p q} \psi\right) \equiv \sqrt{-g} \bar{\chi} \gamma^{\mu}\left(\partial_{\mu} \chi+\frac{1}{4} \omega_{\mu p q} \gamma^{p q} \chi+\frac{1}{24} H_{\mu p q} \gamma^{p q} \chi\right)
$$

The second equality, or ' $\equiv$ ', holds up to the Riemannian reduction of $\tilde{\partial}^{\mu} \equiv 0$, and a field redefinition of the fermion, $\chi \equiv 2^{-\frac{1}{4}} e^{-\phi} \psi$, which couples to the Riemannian vierbein and $B$-field, as if fundamental string. That is to say, dilaton $\phi$ is dark to the fermion, $\chi \cdot{ }^{4}$ Although quantum field theory is supposed to describe "point-like" particles, the Dirac spinor $\chi$ seems to remember its stringy origin.

From Stringy Gravity point of view, $H$-flux is part of the torsionless connection, and the stringy Christoffel symbol (10) cannot be transformed point-wise to vanish by doubled diffeomorphisms. That is to say, locally inertial (normal) frames do not exist in Stringy Gravity: the Equivalence Principle is broken for a string. This should not be a surprise, since string is an extended object, subject to "tidal force", or $H$-flux. It is also consistent with the absence of a four-indexed "Riemann" curvature in Stringy Gravity [22, 28]. Eq.(12) then implies the violation of the Equivalence Principle for fermions (c.f [29] or "fifth force"). We continue to discuss the recovery of the principle in section 2.4.

Given the twofold local Lorentz symmetries, one needs to decide the spin group for each fermion in the Standard Model after preparing two sets of gamma matrices. An open question, which could be answered experimentally, is whether the spin groups of quarks and leptons are different (or not) [27].

\subsection{Uroboros solution to the Dark Matter and the Dark Energy problems}

Motivated by the coupling of the Standard Model to the closed string massless sector, such as (12), the most general, asymptotically flat, spherically symmetric vacuum solution to $D=4$ Stringy Gravity was studied in [30] (see also [31]). The solution lets the stringy Einstein tensor vanish identically, $G_{A B}=0$, and therefore corresponds to the stringy analogue of the Schwarzschild solution to GR. Yet, it possesses three free parameters: not only mass, $M$ (measurable at infinity), but also, say $H$-flux and dilaton charge. All of them ought to reflect the intrinsic properties of the implicitly assumed

\footnotetext{
${ }^{3}$ Ensuring the twofold spin groups in the maximally supersymmetric DFT [25] and the doubled-yet-gauged Green-Schwarz superstring action [20], the conventional IIA and IIB theories are unified into a single unique theory that is chiral with respect to both spin groups. The distinction of IIA and IIB then refers to two different types of (Riemannian) 'solutions' rather than 'theories'.

${ }^{4}$ On the other hand, gauge bosons couple to $g_{\mu \nu}$ and $\phi$ only: $H$-flux is dark to the gauge bosons.
} 
matter localized at the center, which deserve, combined with (12), further explorations, possibly in connection with 'energy condition' in GR.

The generic spherical solution would be regarded to feature a naked singularity, if it were interpreted from GR point of view, c.f. (2). But, strictly within the framework of Stringy Gravity, it may be non-singular: all the stringy curvature tensors are trivial. Inevitably, the notion of singularity depends on the differential geometry in use. Investigation into the geodesic completeness is of interest. ${ }^{5}$

Analysis of the circular geodesic around the center in 'string frame' - to be justified in section 2.4 below - shows that the rotation curve generically features a maximum and thus non-Keplerian over a finite range, while becoming asymptotically Keplerian toward infinity [30]. Specifically, with appropriately tuned $H$-flux and dilaton charge, in terms of $R /(M G)$, i.e. the dimensionless radial variable normalized by mass, Stringy Gravity agrees with GR becoming Keplerian for large $R /(M G) \gtrsim 10^{8}$, but modifies it at shorter distance, $R /(M G) \lesssim 2 \times 10^{6}$ (c.f. MOND [32]). At far shorter distance, gravitational force can be even repulsive. These may solve the dark matter and energy problems which are essentially based on small $R /(M G)$ observations: long distance divided by much heavier mass.

Namely, the self-interaction of the closed string massless sector makes Stringy Gravity modify $G R$ at 'short' distance in terms of $R /(M G)$, such that it may explain the observed galaxy rotation curves, hence solve the dark matter problem. Further, the repulsive force at far shorter distance can be responsible for the accelerating expansion of the Universe, and thus solve the dark energy problem.

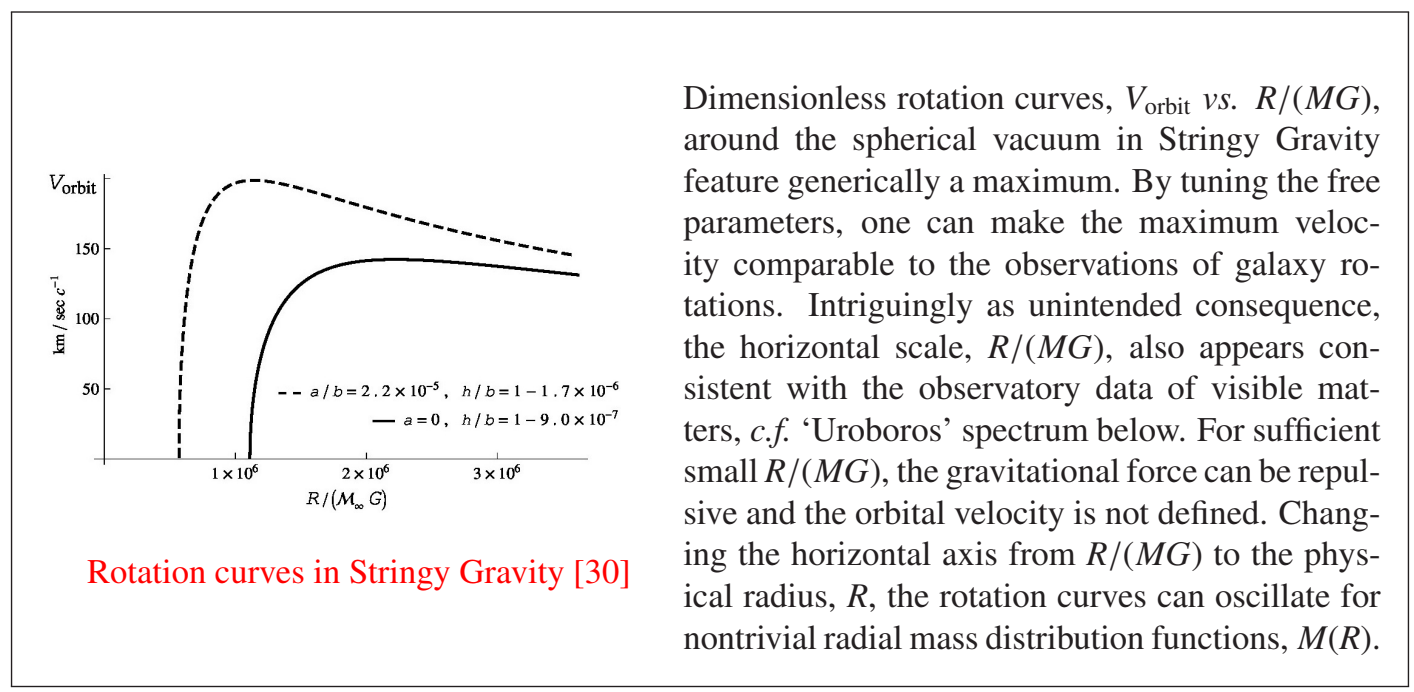

\begin{tabular}{|c|c|c|c|c|c|c|c|c|c|}
\hline & $\begin{array}{c}\text { Electron } \\
(R \simeq 0)\end{array}$ & Proton & $\begin{array}{c}\text { Hydrogen } \\
\text { Atom }\end{array}$ & $\begin{array}{c}\text { Billiard } \\
\text { Ball }\end{array}$ & Earth & $\begin{array}{c}\text { Solar System } \\
\left(1 \mathrm{AU} / M_{\odot} G\right)\end{array}$ & $\begin{array}{c}\text { Milky Way } \\
\text { (visible) }\end{array}$ & $\begin{array}{c}\text { Galaxy } \\
\text { Cluster }\end{array}$ & $\begin{array}{c}\text { Universe } \\
\left(M \propto R^{3}\right)\end{array}$ \\
\hline$R /(M G)$ & $0^{+}$ & $7.1 \times 10^{38}$ & $2.0 \times 10^{43}$ & $2.4 \times 10^{26}$ & $1.4 \times 10^{9}$ & $1.0 \times 10^{8}$ & $1.5 \times 10^{6}$ & $\sim 10^{5}$ & $0^{+}$ \\
\hline
\end{tabular}

'Uroboros' spectrum of the dimensionless radial variable normalized by mass in natural units. Observing stars and galaxies far away, we can probe deep into the short distance natrue of GRAVITY.

\footnotetext{
${ }^{5}$ c.f. both (1) and (17), as well as cosmic censorship à la Penrose.
} 


\subsection{Proper distance in doubled-yet-gauged spacetime: Justification for String Frame}

Shifting gears, we now discuss how to define the proper distance in doubled-yet-gauged spacetime. First of all, we note that the usual infinitesimal one-form, $\mathrm{d} x^{A}$, is neither doubled diffeomorphism covariant, $\delta\left(\mathrm{d} x^{A}\right)=\mathrm{d} x^{B} \partial_{B} V^{A} \neq \mathrm{d} x^{B}\left(\partial_{B} V^{A}-\partial^{A} V_{B}\right)$, (7), (8), nor coordinate gauge symmetry invariant, $\mathrm{d} \Delta^{A}=\mathrm{d} x^{B} \partial_{B} \Delta^{A} \neq 0$, (5). Thus, the naive contraction with the DFT-metric, $\mathrm{d} x^{A} \mathrm{~d} x^{B} \mathcal{H}_{A B}$, cannot give any sensible definition of the proper length. To cure the problem, one needs to gauge $\mathrm{d} x^{A}$ explicitly, introducing a connection, $\mathcal{A}^{A}$, which should satisfy the same property as the coordinate gauge symmetry generator, $\Delta^{A}(5)$,

$$
\mathrm{D} x^{A}:=\mathrm{d} x^{A}-\mathcal{A}^{A}, \quad \mathcal{A}^{A} \partial_{A}=0 .
$$

Provided the connection transforms appropriately, $\mathrm{D} x^{A}$ becomes a well-behaved vector of stringy geometry, meaning doubled diffeomorphism covariant and coordinate gauge symmetry invariant [18]. We propose then to define the proper distance in doubled-yet-gauged spacetime by a path integral [33],

$$
\left\|x_{1}, x_{2}\right\|:=-\ln \left[\int \mathcal{D} \mathcal{A} \exp \left(-\int_{1}^{2} \sqrt{\mathrm{D} x^{A} \mathrm{D} x^{B} \mathcal{H}_{A B}}\right)\right]
$$

By letting $\tilde{\partial}^{\mu} \equiv 0$ and $\mathcal{A}^{A} \equiv\left(A_{\mu}, 0\right)$, we get $\mathrm{D} x^{A} \equiv\left(\mathrm{d} \tilde{x}_{\mu}-A_{\mu}, \mathrm{d} x^{\nu}\right)$. That is to say, only the half of the doubled coordinates, i.e. $\tilde{x}_{\mu}$ directions, are gauged. Furthermore, with the Riemannian parametrization of the DFT-metric, we get

$$
\mathrm{D} x^{A} \mathrm{D} x^{B} \mathcal{H}_{A B} \equiv \mathrm{d} x^{\mu} \mathrm{d} x^{v} g_{\mu v}+\left(\mathrm{d} \tilde{x}_{\mu}-A_{\mu}+\mathrm{d} x^{\rho} B_{\rho \mu}\right)\left(\mathrm{d} \tilde{x}_{v}-A_{v}+\mathrm{d} x^{\sigma} B_{\sigma v}\right) g^{\mu \nu} .
$$

Thus, after integrating out the auxiliary connection, $A_{\mu}$, our proposal (14) reduces, at least classically, to the conventional proper distance in Riemannian geometry, $\int_{1}^{2} \sqrt{\mathrm{d} x^{\mu} \mathrm{d} x^{\nu} g_{\mu \nu}}$, which is certainly independent of the gauged $\tilde{x}_{\mu}$ coordinates:

$$
\left\|x_{1}^{A}, x_{2}^{A}\right\|=\left\|x_{1}^{\mu}, x_{2}^{\mu}\right\| .
$$

In this way, indeed the formula (14) measures the distance between two 'gauge orbits'

The exponent in (14) sets immediately the action for a point particle propagating in the doubledyet-gauged spacetime, or its square root free einbein formulation [30, 34]. As a consequence, after the auxiliary connection being integrated out, the point particle couples to $g_{\mu \nu}$ only. That is to say, both $\phi$ and $B_{\mu \nu}$ are dark to particles: they are hard to detect by any experiment based on particle concept. Further, the point particle follows the geodesic defined not in the Einstein frame but in the string frame. Namely, the Equivalence Principle is restored in the string frame for a point particle. This preferred choice of the frame is all due to the fundamental symmetries of Stringy Gravity.

The above path integral definition of the proper length can be easily generalized to (NambuGoto type) area and volume, which in turn gives the doubled-yet-gauged actions for string or GreenSchwarz superstring $[18,20]$. In particular, with auxiliary worldsheet metric, $h_{i j}$, and the stringy Christoffel symbol (10), the Euler-Lagrange equations of the string action reduce, upon Riemannian backgrounds (c.f. non-Riemannian [21]), to

$$
\frac{1}{\sqrt{-h}} \partial_{i}\left(\sqrt{-h} \mathcal{H}_{A B} D^{i} x^{B}\right)+\Gamma_{A B C}\left(\bar{P}^{B}{ }_{D} D_{i} x^{D}\right)\left(P^{C}{ }_{E} D^{i} x^{E}\right)=0
$$

which can be viewed as the stringy analogue of the point particle geodesic equation (1). 


\section{Acknowledgements}

This note is based on series of works by the author in collaborations with Imtak Jeon, Kanghoon Lee, Soo-Jong Rey, Yuho Sakatani, Yoonji Suh, Wonyoung Cho, Jose Fernández-Melgarejo, Woohyun Rim, Sung Moon Ko, Charles Melby-Thompson, Rene Meyér, Minwoo Suh, Kang-Sin Choi, Kevin Morand and Xavier Bekaert, as well as discussions with Sang-Hyeon Ahn, David Berman, Chris Blair, Robert Brandenberger, Martin Cederwall, Jaewon Lee, Chan Park, Hyun Seok Yang and Sang Heon Yi. The author wishes to thank them all. This work was supported by the National Research Foundation of Korea through the Grant NRF-2016R1D1A1B01015196.

\section{References}

[1] T. H. Buscher, “A Symmetry of the String Background Field Equations,” Phys. Lett. B 194 (1987) 59.

[2] T. H. Buscher, "Path Integral Derivation of Quantum Duality in Nonlinear Sigma Models," Phys. Lett. B 201 (1988) 466.

[3] M. J. Duff, "Duality Rotations in String Theory," Nucl. Phys. B 335 (1990) 610.

[4] A. A. Tseytlin, "Duality Symmetric Formulation of String World Sheet Dynamics," Phys. Lett. B 242 (1990) 163.

[5] A. A. Tseytlin, "Duality symmetric closed string theory and interacting chiral scalars," Nucl. Phys. B 350 (1991) 395.

[6] C. M. Hull, "A Geometry for non-geometric string backgrounds," JHEP 0510 (2005) 065.

[7] C. M. Hull, "Global aspects of T-duality, gauged sigma models and T-folds," JHEP 0710 (2007) 057.

[8] C. M. Hull, "Doubled Geometry and T-Folds," JHEP 0707 (2007) 080 [hep-th/0605149].

[9] W. Siegel, "Two vierbein formalism for string inspired axionic gravity," Phys. Rev. D 47 (1993) 5453 [hep-th/9302036].

[10] W. Siegel, "Superspace duality in low-energy superstrings," Phys. Rev. D 48 (1993) 2826 [hepth/9305073].

[11] C. Hull and B. Zwiebach, "Double Field Theory," JHEP 0909 (2009) 099 [arXiv:0904.4664 [hep-th]].

[12] C. Hull and B. Zwiebach, "The Gauge algebra of double field theory and Courant brackets," JHEP 0909 (2009) 090 [arXiv:0908.1792 [hep-th]].

[13] O. Hohm, C. Hull and B. Zwiebach, "Generalized metric formulation of double field theory," JHEP 1008 (2010) 008 [arXiv:1006.4823 [hep-th]].

[14] G. Aldazabal, D. Marques and C. Nunez, "Double Field Theory: A Pedagogical Review," Class. Quant. Grav. 30 (2013) 163001 doi:10.1088/0264-9381/30/16/163001 [arXiv:1305.1907 [hep-th]].

[15] D. S. Berman and D. C. Thompson, "Duality Symmetric String and M-Theory,” Phys. Rept. 566 (2014) 1 doi:10.1016/j.physrep.2014.11.007 [arXiv:1306.2643 [hep-th]].

[16] O. Hohm, D. Lüst and B. Zwiebach, "The Spacetime of Double Field Theory: Review, Remarks, and Outlook," Fortsch. Phys. 61 (2013) 926 doi:10.1002/prop.201300024 [arXiv:1309.2977].

[17] J. H. Park, "Comments on double field theory and diffeomorphisms," JHEP 1306 (2013) 098 [arXiv:1304.5946 [hep-th]].

[18] K. Lee and J. H. Park, "Covariant action for a string in doubled-yet-gauged spacetime," Nucl. Phys. B 880 (2014) 134 [arXiv:1307.8377 [hep-th]].

[19] S. M. Ko, C. Melby-Thompson, R. Meyer and J. H. Park, "Dynamics of Perturbations in Double Field Theory \& Non-Relativistic String Theory,” JHEP 1512 (2015) 144 [arXiv:1508.01121 [hepth]]. 
[20] J. H. Park, “Green-Schwarz superstring on doubled-yet-gauged spacetime,” JHEP 1611 (2016) 005 [arXiv:1609.04265 [hep-th]].

[21] K. Morand and J. H. Park, "Classification of non-Riemannian doubled-yet-gauged spacetime," arXiv:1707.03713 [hep-th].

[22] I. Jeon, K. Lee and J. H. Park, “Stringy differential geometry, beyond Riemann,” Phys. Rev. D 84 (2011) 044022 [arXiv:1105.6294 [hep-th]].

[23] J. H. Park, S. J. Rey, W. Rim and Y. Sakatani, "O(D, D) covariant Noether currents and global charges in double field theory," JHEP 1511 (2015) 131 [arXiv:1507.07545 [hep-th]].

[24] M. J. Duff, “Hidden String Symmetries?," Phys. Lett. B 173 (1986) 289.

[25] I. Jeon, K. Lee, J. H. Park and Y. Suh, "Stringy Unification of Type IIA and IIB Supergravities under $N=2 D=10$ Supersymmetric Double Field Theory," Phys. Lett. B 723 (2013) 245 [arXiv:1210.5078 [hep-th]].

[26] I. Jeon, K. Lee and J. H. Park, "Incorporation of fermions into double field theory," JHEP 1111 (2011) 025 [arXiv:1109.2035 [hep-th]].

[27] K. S. Choi and J. H. Park, "Standard Model as a Double Field Theory," Phys. Rev. Lett. 115 (2015) no.17, 171603 [arXiv:1506.05277 [hep-th]].

[28] O. Hohm and B. Zwiebach, "On the Riemann Tensor in Double Field Theory," JHEP 1205 (2012) 126.

[29] T. D. Lee and C. N. Yang, "Conservation of Heavy Particles and Generalized Gauge Transformations,” Phys. Rev. 98 (1955) 1501.

[30] S. M. Ko, J. H. Park and M. Suh, "The rotation curve of a point particle in stringy gravity," arXiv:1606.09307 [hep-th]. To appear in JCAP.

[31] C. P. Burgess, R. C. Myers and F. Quevedo, "On spherically symmetric string solutions in fourdimensions,” Nucl. Phys. B 442 (1995) 75 [hep-th/9410142].

[32] M. Milgrom, "A Modification of the Newtonian dynamics as a possible alternative to the hidden mass hypothesis," Astrophys. J. 270 (1983) 365.

[33] J. H. Park, "Green-Schwarz superstring and Stringy Gravity in doubled-yet-gauged spacetime," talk at Banff International Research Station, http://www.birs.ca/events/2017/5-day-workshops/ 17w5018/videos/watch/201701231429-Park.html.

[34] C. D. A. Blair, "Particle actions and brane tensions from double and exceptional geometry," arXiv:1707.07572 [hep-th]. 